3017

Regulatory Science in Translational and Regenerative Medicine Biomedical Education: A Pilot Course

Alexandra Joelle Greenberg-Worisek, PhD, MPH${ }^{1}$, Luz Cumba-Garcia ${ }^{1}$, Nilay Shah, PhD ${ }^{1}$ and Anthony Windebank, MD ${ }^{1}$ ${ }^{1}$ Mayo Clinic

OBJECTIVES/SPECIFIC AIMS: INTRODUCTION: Many of the innovative therapies used in regenerative medicine, such as additive manufacturing and stem cell engineering, rely on novel technologies and techniques for which standards for safety, efficacy, and quality have not been fully explored and established. As these therapies and technologies develop at a rapid pace, there is a need for the development of scientifically-based metrics to assess whether new treatments are effective and safe for clinical translation. Research and development of such standards is known as "regulatory science," and is needed by the United States Food and Drug Administration (FDA) to support the creation of the evidence-based guidances and regulations that are used in review of product submissions. As outlined by the FDA's description of their Centers for Excellence in Regulatory Science and Innovation (CERSIs) and the Association for Clinical and Translational Science's (ACTS) Regulatory Science Working Group, here is a need to train investigators and clinicians to conduct regulatory science research to support successful clinical translation of regenerative treatments. OBJECTIVE: To develop a course to expose scholars to regulatory science concepts, to empower them to apply these concepts to their personal areas of research, and to challenge them to engage in the dialogue surrounding regulatory science on a national level. METHODS/STUDY POPULATION: METHODS: The "Introduction to Regulatory Science" course was developed jointly by the Yale-Mayo CERSI and the Mayo Clinic Center for Clinical and Translational Science (CCaTS) in 2017 as a didactic in-person survey course. The course objectives included exposing scholars to the ideas of regulatory science and affairs; reviewing the FDA's Priority Areas for Advancing Regulatory Science; and determining what safety, quality, and efficacy concerns may need to be addressed when using new technologies, such as those used in regenerative medicine research. To meet these intended learning objectives, the course addressed one FDA Priority area each week, with a team of experts providing one-hour of lecture and discussion each class session. Regenerative medicine-related topics included a bioethics of stem cell therapy development, evaluation of additive manufacturing as an emerging technology, and the application of cGMPs to the manufacture of new therapies. Assignments and assessments included a quiz each week, which served as a knowledge check of that week's lecture content, and a final paper analyzing regulatory concerns associated with a technology or product of the scholar's choosing. RESULTS/ANTICIPATED RESULTS: RESULTS: The course was first delivered as an elective in 2017, with an enrollment of 8 scholars and 3 auditors. Scholars enrolled included 7 trainees and established investigators from Mayo Clinic Rochester and one from Arizona. Of the 8 scholars enrolled, $7 \mathrm{com}$ pleted the post-course survey. Scholars strongly agreed that the "course objectives were met" (7/7) and that the "course was well worth the effort I put into it" (7/7). Five scholars stated that they learned "a lot" during the course; two said they learned "an incredible amount." Scholars unanimously gave the course a grade of "A." Qualitative feedback was positive, indicating that the team-taught and in-person course design choices were highlights for trainees. The course is currently in its second delivery (2018), with an enrollment of 16 scholars across Mayo Clinic Rochester, Arizona, and Florida.
DISCUSSION/SIGNIFICANCE OF IMPACT: CONCLUSIONS: The developed course was successfully piloted and well-received. Scholars reported that they agreed that the course aims were achieved, and indicated that they would like to see additional coursework to continue to learn how to engage in regulatory science. Next steps include utilizing course feedback to iterate on the current course, expanding course delivery to include scholars at the Yale site of the Yale-Mayo CERSI, and the creation of a second course.

3351

\section{Research training and career development in junior investigators at a multi-institutional CTSA}

Jachael Gardner ${ }^{1}$, Jim Morrison, Terry Nakazono and

Pamela Davidson

${ }^{1}$ David Geffen School of Medicine at UCLA

OBJECTIVES/SPECIFIC AIMS: The study aims to understand the characteristics of junior investigators who are supported by the CTSI, their knowledge of CTSI services and resources, as well as the perceived effectiveness of CTSI research training and career development. The primary outcome is scientific productivity that enhances career development and promotion. The secondary outcome is to inform and improve CTSA research training and career development, not only for the UCLA CTSI hub but also for the CTSA Program nationally. METHODS/STUDY POPULATION:. The study sample includes post-doctoral and junior investigators who have received UCLA CTSI support between 2011 and 2017 $(n=319)$. These junior investigators conduct research at our four partner sites (UCLA-Westwood, Cedars-Sinai Medical Center, The Los Angeles Biomedical Research Institute at Harbor-UCLA Medical Center, Charles R. Drew University of Medicine and Science). The CTSI administered the LSAS and the Career Development module in 2018 using Qualtrics, a subscription software for collecting and analyzing survey data. In order to reduce the burden on the survey respondents, the survey team pre-populated their previously reported publication in the Qualtrics survey system. Qualtrics sends customized email invitations containing a link to start the LSAS survey and subsequently automated sequences of reminder messages for non-responders and partial completers. The survey team initiated telephone call-backs and engaged partner site leaders to achieve a high response rate. RESULTS/ANTICIPATED RESULTS:. Preliminary results show a response rate of $83 \%(n=264)$. Respondents include K-to- $\mathrm{R}$ workshop participants $(\mathrm{n}=192)$, core voucher coinvestigators $(\mathrm{n}=38)$, Training Program in Translational Science (TPTS) trainees $(\mathrm{n}=82)$, and junior investigators who participated in two or more of these activities $(n=48)$. Trainee characteristics include degree, gender, and partner site location. The distribution of advanced degrees among the junior investigators include: $\mathrm{PhD}$ (29\%), MD (55\%), MD/PhD (13\%), or other degree (3\%). Fortyfour percent $(44 \%)$ respondents are male and $56 \%$ are female. Seventy-two percent (72\%) have a primary appointment at UCLAWestwood, while $28 \%$ have academic appointments at the partner institutions. Twenty-five percent (25\%) received CTSI research training and $49 \%$ received CTSI career development support. Regarding scientific productivity, $58 \%$ reported having at least one peer-reviewed publication and $29 \%$ reported subsequent grant funding. DISCUSSION/SIGNIFICANCE OF IMPACT:. We are in the process of identifying the predictors of scientific productivity and the characteristics of the junior investigators most significantly correlated with productivity using multiple logistic regression analysis. We will analyze qualitative responses to examine the facilitators 